\title{
El juego como estrategia didáctica para el desarrollo motriz
}

\author{
Zoraida Rocío Manrique Chávez \\ zmanriquec@unia.edu.pe \\ Universidad Nacional Intercultural de la Amazonía \\ Anthony Rosseau Flores Espinoza \\ aflorese@unia.edu.pe \\ Universidad Nacional Intercultural de la Amazonía \\ Alejandro Manuel Ecos Espino \\ aecose@unam.edu.pe \\ Universidad Nacional de Moquegua \\ Rosario Mercedes Aguilar Melgarejo \\ rosario.aguilar@unh.edu.pe \\ Universidad Nacional de Huancavelica \\ Rosa Manrique Chávez \\ rochi_2911@hotmail.com \\ Unidad de Gestión Educativa Local \\ Olga Isabel Carbajal Guerrero \\ ocarbajalg@unia.edu.pe \\ Universidad Nacional Intercultural de la Amazonía \\ Ucayali- Perú
} RESUMEN

Esta investigación tuvo como propósito evaluar la influencia que ejerce el juego considerada estrategia didáctica en el desarrollo motor de los niños y niñas pertenecientes a la I. E. I. N 575 de la localidad de Atalla de la provincia de Huancavelica. Para tal propósito, se recurrió al Test De Desarrollo Motor Grueso (TGMG -2), aplicado a 15 niños y niñas con edades entre 3 y 4 años, antes y después de la ejecución de una estrategia didáctica basada en juegos. Dicho instrumento evalúa el desarrollo relacionado a la habilidad de locomoción y de control de objetos. Los resultados obtenidos muestran que, usando un nivel de significancia de 0,05, la aplicación de la estrategia didáctica basada en juegos mejora de manera significativa el desarrollo motor en relación a las habilidades de locomoción y control de objetos, ubicando a más del $90 \%$ de los niños por encima del nivel pobre de desarrollo motor.

Palabras clave: desarrollo motor; juego; estrategia didáctica. 


\title{
The game as a didactic strategy for motor development
}

\begin{abstract}
This research had as objective to evaluate the influence of the game as a didactic strategy in the motor development of the children of the Educational Institution of the locality of Atalla of the province of Huancavelica. (TGMG-2) before and after the application of games as a didactic strategy. This instrument evaluates the development related to the ability of locomotion and control of objects. The results obtained that a level of relevance of 0.05 , the use of the game as didactic strategy improves of important way the motor development in relation to the abilities of the locomotive and the control of objects, placing more than $90 \%$ of the Children above the level of motor development.
\end{abstract}

Keywords: motor development; game; didactic strategy

Artículo recibido: 10. Junio. 2021 Aceptado para publicación: 16. Julio. 2021

Correspondencia: zmanriquec@unia.edu.pe Conflictos de Interés: Ninguna que declarar 


\section{INTRODUCCIÓN}

Al inicio de sus años de vida, la motricidad y el juego representan elementos primordiales para el desarrollo del niño; sin embargo, en el entorno familiar y en muchas instituciones educativas no constituyen lo suficientemente importante. La motricidad permite que el niño desarrolle su personalidad, así como la mejora de sus aprendizajes, ayudándole a ser más feliz, equilibrado y autónomo. Bilbao, Corres y Urdampilleta (2012). Las interacciones que el niño ha tenido, ya sea dentro de su contexto familiar como en otros espacios, así como el inicio de la vida escolar se encuentra vinculado a su proceso de aprendizaje. Por lo tanto, el aprendizaje de los niños en su primera infancia no solo es responsabilidad de la escuela. Jesús y Jiménez (2015).

Gutiérrez (2009), afirma que, para el desarrollo de las componentes motor, cognitivo, afectivo y social de la personalidad, un elemento importante es la psicomotricidad. A través de ella, el niño podrá conocer su cuerpo y en consecuencia el mundo que está a su alrededor. Por lo tanto, se hace importante llevar a cabo, cuentos, juegos y canciones cuando el niño tiene sus primeros años de vida.

Por lo tanto, debemos resaltar lo importante que resulta el desarrollo de la motricidad en los inicios de vida del niño, que debe generar que la familia participe de manera activa. En tal sentido, se hace necesario desarrollar alternativas pedagógicas que busquen mejorar las habilidades motoras de nuestros niños para que de esta manera coadyuvemos a su desarrollo integral. En tal sentido, el juego resulta una posibilidad posible a investigar para evaluar de qué manera puede ayudar a desarrollar la motricidad.

Para abordar estos puntos, se ha planteado esta investigación que busca evaluar la influencia de una estrategia didáctica basada en el juego, en la evolución y crecimiento de la motricidad en niños de la I. E. $N^{\circ} 575$ de la localidad de Atalla de la Provincia de Huancavelica.

El juego es un componente primordial en la formación integral de los niños, ya que permite el desarrollo de éstos, tanto a nivel cognitivo, así como en su faceta social y personal. Por medio del juego, los niños se expresan con mayor libertad, desarrollan su imaginación, pueden crear su autonomía y su identidad. De 
igual forma, por medio del juego los niños pueden mejorar la convivencia con otros niños, como también pueden desarrollar sus capacidades motoras. Por tales motivos, en la educación inicial se pretende motivar al niño a que exprese sus pensamientos, sus sentimientos y sus emociones mediante su cuerpo, haciendo una integración con otras formas de lenguajes como: el movimiento, la imitación, el sonido y el ritmo Calderón (2012); en tal sentido, el desarrollo de esta investigación es importante porque constituirá un aporte a la consecución de dicho objetivo.

Por otro lado, a través de este trabajo se busca aportar una alternativa metodológica a los docentes del nivel basada en el juego, de tal manera que permita la evolución de la componente motora de los niños. Además, el análisis teórico llevado a cabo permitirá aportar mayores argumentos al fundamento teórico del juego como alternativa didáctica, por lo que el diseño metodológico construido en este trabajo servirá de guía para el desarrollo de otras investigaciones que busquen la generación de nuevas posibilidades didácticas a llevarse a cabo dentro del aula.

Espinoza (2010) define al juego como: "una actividad fundamental para las personas en el contexto social, porque a través de él se puede experimentar ciertos comportamientos sociales; además, constituye un medio útil para la adquisiciòn y fortalecimiento de capacidades intelectuales, motoras o afectivas. Para todo ello, la organización del tiempo y espacio necesario para efectuar estas actividades es necesario, las cuales deben realizarse de manera gustosa y placentera"

Patiño y otros (2012), plantean que: "la psicomotricidad se relaciona directamente con el cuerpo y sus experiencias". Así, se considera como una técnica que facilita "la manifestación las capacidades relativas al movimiento, del propio cuerpo, descubriendo su entorno y de los otros ". Abbadie (1976).

La capacidad que adquiere el niño para desplazarse y para efectuar movimientos disociados se va desarrollando de acuerdo como el niño va madurando de manera neurológica y motora. Defontaine (1981), plantea que la educación psicomotriz es "una reestructuración, de sí mismo, una reflexión sobre sí mismo, una reintegración social, una reexpresión tomando como referencia el denominador corporal común". 
Según Vayer (1984), "la educación psicomotriz asume la problemática de la educación por medio del movimiento". Es decir, este tipo de educación debe pensarse de acuerdo a los años que tenga el niño, las motivaciones del niño y de su unidad, favoreciendo de este modo el desarrollo de su personalidad.

Según Jiménez (2016): "El niño tiende a mejorar su relación con las personas y objetos de su entorno, así como sus capacidades para desplazarse, en la medida que controla su cuerpo. En consecuencia, el elemento primordial de contacto con su entorno es su propio cuerpo. La manipulación, análisis y síntesis del mundo exterior por parte del niño será realizado a través de su actividad corporal, lo cual le permitirá reforzar el desarrollo de estas capacidades en su etapa adulta".

\section{MÉTODO}

El estudio está enmarcado en una investigación aplicada, pues trata de poner en evaluación una estrategia didáctica en base a juegos a fin de evaluar sus efectos sobre el desarrollo motor en los niños de la I. E. $\mathrm{N}^{\circ} 575$ de la localidad de Atalla de la Provincia de Huancavelica. El estudio dio respuesta a un problema de carácter teórico sobre las variaciones de cierto modelo de manera tal que su orientación es la descripción y la explicación Sánchez y Reyes (2006)

Se trabajó con un diseño cuasi experimental, con un solo grupo. Se evaluó con una población conformada por 50 niños de la I. E. I. N ${ }^{\circ} 575$ de la localidad de Atalla. Con una muestra que estuvo compuesta por la totalidad de niños pertenecientes al salón de 3 y 4 años de la I. E. $N^{\circ} 575$ de la localidad de Atalla de la Provincia de Huancavelica.

En este estudio se aplicó el Test de Desarrollo Motor Grueso (TGDM-2) elaborado por Ulrich (2000). A través de este instrumento se obtiene una medida referencial sobre la motricidad gruesa, la cual podrá ser utilizado por educadores, kinesiólogos, psicólogos, generalistas, fisioterapeutas y especialistas. Este instrumento permite identificar los alumnos que tienen una incorrecta evolución de sus habilidades motrices básicas comparadas con el resto de sus compañeros a fin de ser elegidos para acogerse a una educación diferenciada. En tal sentido, con este test se busca determinar un coeficiente 
para describir la evolución de las habilidades motoras que se apoyan en la coordinación del cuerpo del niño.

La prueba se administra de manera individual, así como en pequeños grupos. Las puntuaciones obtenidas se registran de forma simultáneamente con la ejecución y la terminación de tareas. Cada criterio de desempeño en cada habilidad motora, las puntuaciones pueden ser: 0 (pero si falla un intento) o 1 (cuando se finaliza con éxito un intento). Estas puntuaciones se aplican en cada criterio de rendimiento para los ensayos que se realizan en cada habilidad. La suma de estas puntuaciones generan un índice de habilidad en relación de cada componente. Luego de sumar las puntuaciones en las seis tareas de movimiento en cada dominio, se obtendrá una puntuación sub-prueba en relación a las habildades locomotoras y las de control de objetos. Los materiales para ejecutar el test se pueden encontrar en cualquier centro escolar, lo que hace la aplicación mucho más fácil.

El tratamiento de la información se efectuó a través de la estadística descriptiva. Luego de cuantificar las puntuaciones por medio de los instrumentos de investigación, la Prueba de Shapiro permitió evaluar el nivel de normalidad de los datos. El Coeficiente de Pearson, a un nivel de significación de 0,05, se usó en la contrastación de las hipótesis y evaluó la relación que existe entre las variables. También, la Prueba de Homogeneidad de varianza que permitió hacer uso de la Prueba $\mathrm{T}$ de Students con la finalidad de realizar la comparación de las medias encontradas en el Pre Test y el Post Test a fin de evaluar el efecto de la estrategia didáctica basada en juegos, en la evolución psicomotora de los niños de la I. E. $\mathrm{N}^{\circ}$ 575 de la localidad de Atalla.

Se hizo el análisis de varianza para demostrar que los resultados obtenidos son diferentes a pretest y postest como es nuestro caso. Durante la contrastación de hipótesis, se hizo uso de un nivel de significancia de 0,05. Toda la información mediante del paquete estadístico SPSS 24.

\section{RESULTADOS}

A continuación, se muestran los resultados encontrados sobre el nivel de desarrollo motor antes y después de la aplicación del juego como estrategia didáctica. 


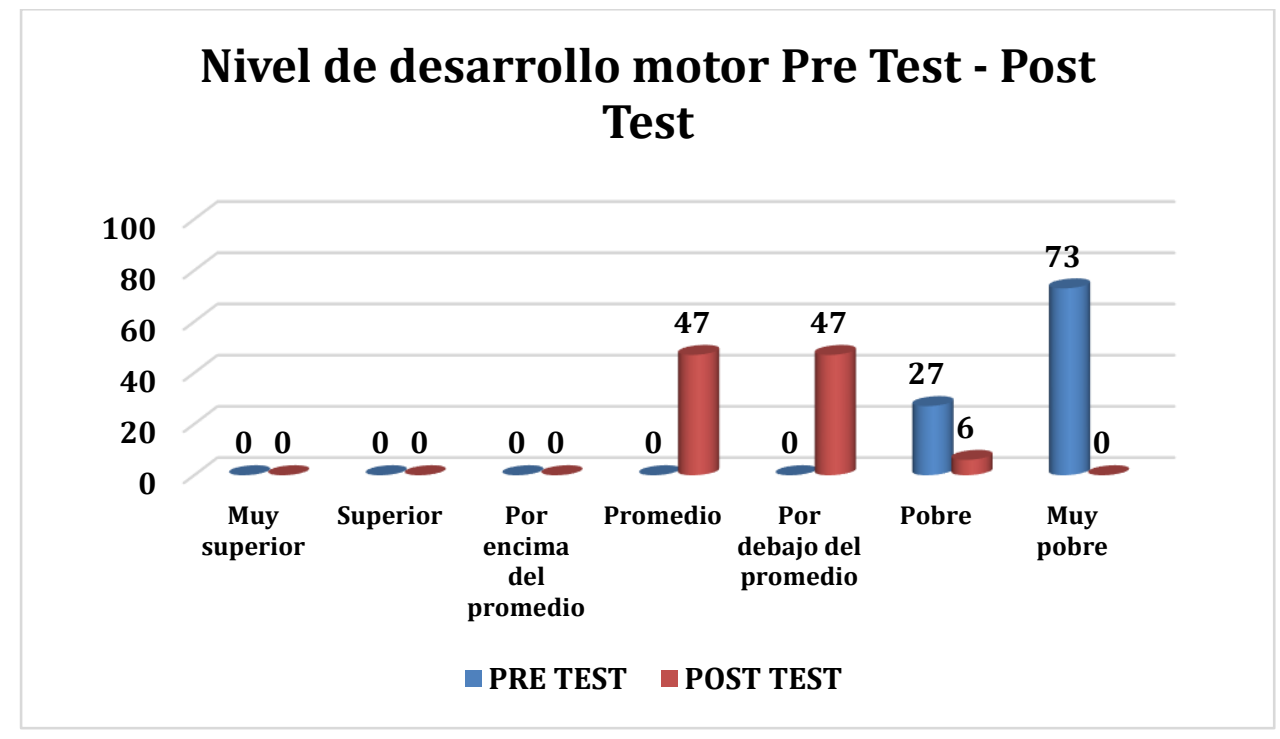

Gráfico 1. Nivel de desarrollo motor en el Pre y Post Test

El gráfico anterior muestra que el 100\% de los niños evaluados en el pre test se encuentra por debajo del promedio en relación al desarrollo motor. El 27\% presenta un pobre nivel mientras que un significativo $73 \%$ se encuentra en un nivel pobre de desarrollo motor, antes de la aplicación de los juegos con un pobre desarrollo motor.

Después de la aplicación del juego como estrategia didáctica, podemos observar la existencia de mejoras en cuanto a este nivel con relación al pre test. Solo el $6 \%$ se mantiene en un nivel podre de desarrollo motor. Un 47\% de niños alcanzó el nivel promedio y otro $47 \%$ se mantiene aún por debajo de este promedio, pero sin tener un pobre nivel. Aún con estas mejoras, la totalidad de la muestra no supera el promedio de nivel de desarrollo motor como resultado de que algunas habilidades no han logrado desarrolle completamente.

Tabla 1. Estadísticos descriptivos del nivel de desarrollo motor en el Pre y Post Test

\begin{tabular}{llllll}
\hline Test & N & Mínimo & Máximo & Media & $\begin{array}{l}\text { Desviación } \\
\text { estándar }\end{array}$ \\
\hline Pre & 15 & 60,00 & 73,00 & 67,7 & 3,9 \\
Post & 15 & 74 & 92 & 86.8 & 5.2 \\
\hline
\end{tabular}

Fuente: Elaboración Propia 
En la tabla 1 podemos observar que el nivel desarrollo motor en los niños en el pre test que alcanza en promedio 67.7 puntos, dentro de un rango en la escala de 60 a 73 puntos. También se constata una desviación estándar de 3.9 puntos indicando la existencia una dispersión no tan significativa en relación al promedio. Además, se observa que el nivel desarrollo motor en los niños en el Post Test que alcanza en promedio 86.8 puntos, donde el rango de las puntuaciones oscila entre 74 y 92 puntos. La desviación estándar de 5.2 puntos indica que la dispersión existente no es tan significativa en relación al promedio. Este promedio, ubica a gran parte de la muestra por debajo del promedio del nivel de desarrollo motor.

Estos resultados muestran el escaso desarrollo motor que presentan los niños de nuestra Institución Educativa y la necesidad que existe de buscar alternativas pedagógicas que permitan revertir esta situación y contribuir al desarrollo integral de nuestros niños.

Como señala Cobos (1995), el desarrollo motor tiene como meta, controlar y dominar el propio cuerpo, de manera que el niño tenga la capacidad de hacer uso de todas las potencialidades relacionadas a la acción y la expresión; lo que permite al niño que se vaya construyendo su propia identidad. Esto permite afirmar estas dificultades manifiestas en los niños, no les permite construirse a sí mismos a partir del movimiento, lo cual tendrá una profunda influencia en su en su desarrollo posterior, ya que, en los primeros años de vida, las primeras manifestaciones de comunicación con su entorno son el movimiento, la postura y el tono muscular.

Tabla 2. Prueba de igual de medias según género en el Pre y Post Test

\begin{tabular}{llccccc}
\hline & & \multicolumn{2}{c}{$\begin{array}{c}\text { Prueba de Levene de } \\
\text { calidad de varianzas }\end{array}$} & \multicolumn{2}{c}{$\begin{array}{c}\text { Prueba t para la igualdad } \\
\text { de medias }\end{array}$} \\
\cline { 3 - 7 } & & F & Sig. & T & gl & $\begin{array}{c}\text { Sig. } \\
\text { (bilateral) }\end{array}$ \\
\hline \multirow{2}{*}{ Pre Test } & $\begin{array}{l}\text { Se asumen } \\
\text { varianzas iguales } \\
\text { No se asumen } \\
\text { varianzas iguales }\end{array}$ & 0 & 0.997 & -3.998 & 13 & 0.002 \\
\hline \multirow{2}{*}{ Post Test } & $\begin{array}{l}\text { Se asumen } \\
\text { varianzas iguales } \\
\begin{array}{l}\text { No se asumen } \\
\text { varianzas iguales }\end{array}\end{array}$ & 3.059 & 0.104 & -3.971 & 12.314 & 0.002 \\
\hline
\end{tabular}

Fuente: Elaboración Propia 
En la tabla 2 encontramos que las varianzas en ambos grupos no presentan diferencias estadísticas significativas ya que el valor $\mathrm{p}=0.104$ es mayor al nivel de significancia 0,05 . Esto nos ha permitido evaluar la igualdad de medias encontrándose un valor $\mathrm{p}=0,473 \mathrm{el}$ cual es mayor a 0,05 , por lo tanto, podemos afirmar estadísticamente que existe diferencia entre las medias del nivel de desarrollo motor en niños o niñas de la muestra del presente estudio. Es decir, el nivel de desarrollo motor en las niñas es mejor en comparación con los niños.

\section{DISCUSIÓN}

El juego constituye una actividad importante dentro del desarrollo y crecimiento del niño. Zúgaro (1992) lo considera como el elemento sustancial para la socialización del niño. Froebel (1913) lo considera como el reflejo de la vida interna del niño [...] que le genera placer, gozo y una sensación de satisfacción con uno mismo. Piaget (1896) afirma que en el juego se encuentra todas las oportunidades de paso entre el hacer constructivo y la imaginación creadora, lo cual establece en el niño la continuidad entre el juego y el trabajo.

En tal sentido, las oportunidades de aprendizaje deben componerse de herramientas lúdicas, ya que el juego constituye la manera más espontánea para aprender. Por lo tanto, representa la primera actividad donde el niño hace que la imaginación nazca y se desarrolle, lo cual trae como consecuencia el desarrollo de la creatividad. Además, el juego genera la construcción integral de funciones importantes como el equilibrio, el tono, las conductas perceptivo-motrices así como la lateralidad, permitiendo también el conocimiento y la adaptación a su entorno físico y social. En consonancia a lo propuesto por Pradillo (2007), Mendiara 1999), Madrona y Navarro (2005), se debe construir espacios de acción con actividades motrices que le permitan al niño realizar el juego con libertad y creatividad, permitiendo al maestro ser un animador y generador de situaciones motrices que permitan explorar y descubrir en forma guiada, con consignas abiertas que ayuden a interpretar al niño. En definitiva, se busca la estimulación de conductas sensoriomotores, la mejora de habilidades motrices, así como potenciar la componente cognitiva y la adquisición de tareas, así como el comportamiento de tipo relacional y afectivo. 
Los resultados obtenidos en el pre test, muestra que el nivel de desarrollo motor evidencia las dificultades de los niños para poder realizar actividades relacionadas a la locomoción como saltar, correr y que el desarrollo de estas habilidades es escaso sin distinción de género en la muestra estudiada, así como también en el desarrollo de habilidades relacionadas al control de objetos. Sin embargo, la aplicación del juego como estrategia didáctica ha permitido superar estas primeras dificultades encontradas, tanto a nivel de desarrollo de la locomoción como en el control de objetos. Esto muestra coincidencia con lo planteado por Lara (2013) quien estudio el efecto del juego libre en la evolución de habilidades de tipo social en niños de Educación Inicial, encontrando que estas habilidades se relacionan con el desarrollo motor.

En esta investigación, los resultados evidencian una mejora significativa en cuanto, a las habilidades para saltar obstáculos, correr, desplazamientos a pata coja, galopar, desplazamiento lateral y salto horizontal, así como patear y batear una pelota estática, drible estático, recepcionar, lanzar una pelota por encima del hombro y lanzar por debajo. Esto ha generado que gran parte de los niños hayan superado un pobre nivel de desarrollo motor y se encuentren en un nivel cercano al promedio. Esto coincide con lo encontrado con Gutiérrez (2009) quienes hicieron un estudio sobre el efecto del juego en el desarrollo psicomotor en los niños de tres años, cuyos resultados reflejan que la aplicación del programa fue satisfactorio debido a que el $93 \%$ mejora su coordinación motora grueso.

El niño cuando juega construye relaciones con las demás personas que le ayudan a construir redes de comunicación espontáneas y lazos con los demás personas que conforman su entorno inmediato Camacho (2012).

\section{CONCLUSIONES}

La aplicación de la estrategia didáctica basada en el juego mejora de forma directa y significativa en el desarrollo psicomotor en niños de la I. E. I. $\mathrm{N}^{\circ}$ 575 de la localidad de Atalla de la Provincia de Huancavelica debido a la significancia obtenida en la prueba $T$ de Studenst que demuestra la existencia de diferencias significativas en las medias obtenidas tanto en el pre test como en el post test obteniendo un valor $\mathrm{p}<0.05$, y demostrando que el promedio del post test aumenta significativamente. 
La aplicación de la estrategia didáctica basada en el juego mejora directa y significativamente en el desarrollo de la locomoción en los niños de la I. E. I. $N^{\circ} 575$ de la localidad de Atalla de la Provincia de Huancavelica como consecuencia de la significancia de la prueba $\mathrm{T}$ de Studenst donde se encuentran diferencias significativas entre las medias obtenidas tanto en el pre test como en el post test obteniendo un valor $\mathrm{p}<$ 0,05, demostrando que el promedio obtenido en el post test aumenta significativamente.

La aplicación de la estrategia didáctica basada en el juego mejora de forma directa y significativa en el desarrollo del control de objetos en los niños de la I. E. I. $\mathrm{N}^{\circ} 575$ de la localidad de Atalla de la Provincia de Huancavelica debido a la significancia de la prueba $\mathrm{T}$ de Studenst donde se obtienen diferencias significativas entre las medias obtenida en el pre test y post test obteniendo un valor $\mathrm{p}<0,05$, demostrando que el promedio obtenido en el post test aumenta significativamente

\section{RECOMENDACIONES}

La aplicación de los juegos diseñados para esta investigación ha permitido mejoras significativas en cuanto a las habilidades relacionadas al desarrollo motor grueso, por lo cual, se recomienda replicar los juegos planteados a diferentes poblaciones a fin de ir mejorándolos de manera paulatina por lo cual podríamos seguir coadyuvando al desarrollo motriz en particular y al desarrollo integral de nuestros niños.

Promover dentro de aula con mayor frecuencia, actividades que cooperen a los niños a mejorar sus habilidades para correr, galopar, para realizar desplazamientos a un solo pie, así como efectuar saltos ante obstáculos, saltos horizontales y desplazamientos laterales, de manera tal que coadyuven a el desarrollo de su capacidad locomotora.

Promover dentro de aula con mayor frecuencia, actividades que colaboren a los niños a mejorar sus habilidades para, así como batear o patear una pelota estática, ejecutar dribles estáticos, recepcionar, lanzar una pelota por encima o por debajo del hombro, de manera tal que coadyuven a el desarrollo de su capacidad para controlar objetos. 


\section{REFERENCIAS BIBLIOGRAFICAS}

Abbadie, M. (1976). El niño en el universo del sonido. Buenos Aires: Editorial Kapelusz

Bilbao, L., Corres, U., y Urdampilleta, A. (2012). La importancia de la psicomotricidad en la actividad físico-deportiva extraescolar. Buenos Aires, Argentina: Digital.

Calderón, K. (2012). Análisis de la importancia de la expresión corporal en el desarrollo psicomotor de los niños de 4 y 5 años del Centro de Desarrollo Infantil Divino Niño 1 del Cuerpo de Ingenieros del Ejército de la Ciudad de Quito; Propuesta Alternativa (Tesis de pregrado). Escuela Politécnica del Ejército, Quito, Ecuador.

Camacho, L. (2012). El juego cooperativo como promotor de habilidades sociales en niñas de 5 años (Tesis de pregrado). Pontificia Universidad Católica del Perú, Lima, Perú.

Cobos, P. (1995). El Desarrollo Psicomotor y Sus Alteraciones. Madrid, España: Pirámide.

Defontaine, J. (1978). Manual de reeducation psycomotrice. Barcelona, España: Ed. Médica y Técnica.

Espinoza, G. (2010). Estudio del Juego de niños y niñas de 4 y 5 años y su relación con el desarrollo socio-afectivo (Tesis de pregrado). Universidad de Cuenca, Cuenca, Ecuador.

Froebel, F. (1913). La educación del hombre. Madrid, España.

García, J., y Barruezo, P. (1995). Psicomotricidad y educacióninfantil. Ciencias de la educación preescolar y especial. General Pardiñas. Madrid. España.

Gutiérrez A (2009) La importancia de la educación psicomotriz. Revista digital innovación y experiencias educativas, 24, 1-9.

Gutiérrez, M. (2002). Aprendizaje de valores sociales a través del juego. En: Moreno, J. (2002) Aprendizaje a través del juego. España: Aljibe. p. 52.

Jesús, M. y Jiménez, M. (2015). Influencia del taller Jugando con mi Familia en el Desarrollo Psicomotor de los Niños de 2-3 años de la Institución Educativa Inicial $N^{\circ} 125$ Divino Jesús de Ventanilla - Lima, 2015 (Tesis de Maestría). Universidad Peruana Los Andes, Huancayo, Perú. 
Jiménez, M. (2016). La educación psicomotriz y sus factores a tener en cuenta. Publicaciones $\quad$ Didácticas, Recuperado de: http://publicacionesdidacticas.com/hemeroteca/articulo/067021/articulo-pdf.

Lara, R. (2013). Efecto del programa juego libre para desarrollar habilidades sociales en niños de educación inicial de la institución educativa $n^{\circ} 6099$ Villa el salvador - Lima Perú.

Mendiara, J. (1997). Educación Física y aprendizajes tempranos. Contribución al desarrollo global de los niños de 3 a 6 años y estudio de sus estrategias de aprendizaje en espacios de acción y aventura (Tesis doctoral). Universidad de Zaragoza, Zaragoza, España.

Navarro, V. (2005). El juego motor en educación infantil. Sevilla, España: Wanceulen.

Patiño, W., Gómez, A. Santi, D. y Núñez, O. (2012). El desarrollo psicomotor de los escolares con diagnóstico de retraso mental leve del primer grado, desde la clase de Educación Física. EFDeportes.com, Revista Digital. Buenos Aires, 17 (175)

Piaget, J. (1986): El criterio moral en el niño. Barcelona, España: Fontanela (edición original de 1932).

Pradillo, J. (2007). Higiene, salud y educación física. Aproximación histórica, en ... Pastor Pradillo, J.L. (coord.): Salud, estado de bienestar y actividad física. Aproximación multidimensional. Sevilla: Ed. Wanceulen, 17-34.

Pradillo, J. (2007). Motricidad, ámbitos y técnicas de intervención. Madrid: Universidad de Alcalá.

Sánchez, H. y Reyes, C. (2006). Metodología y Diseños en la Investigación Científica. Lima: Editorial Visión Universitaria.

Ulrich, D.A. (2000). Test of Gross Motor Development: examiner's manual (2a ed.). Austin, Texas: Pro-Ed.

Vayer, P. (1984). Diálogo Corporal -Actividades educativas para niño de 2 a 5 años. Sao Paulo: Editorial Manole.

Vayer, P. (2007) El niño frente al mundo. Barcelona: Científico Médica, 1973. Pastor Pradillo, J.L. Motricidad. Perspectiva psicomotricista de la intervención. Sevilla: Wanceulen. 
Zúgaro, R. E. (1992). Guía para la enseñanza de las danzas folklóricas en la escuela primaria. Buenos Áires, Argentina: La Obra (Información didáctica). 